\title{
Uma Abordagem Para a Organização Automática de Objetos de Aprendizagem em Ambientes Educacionais Ubíquos com Base em Estilos de Aprendizagem
}

\author{
Miller M. Mendes ${ }^{1}$, Vitor C. de Carvalho ${ }^{1}$, Fabiano A. Dorça ${ }^{1}$, Renan G. Cattelan ${ }^{1}$ \\ ${ }^{1}$ Faculdade de Computação - Universidade Federal de Uberlândia (UFU) \\ Uberlândia - MG - Brasil. \\ miller@comp.ufu.br, vitor@mestrado.ufu.br \\ \{renan, fabianodor\} @ufu.br
}

\begin{abstract}
Due to the large amount of learning objects generated from web systems, there is the need to arrange them so that they can be recommended efficiently. Therefore, this work proposes a content clustering for large learning object repositories to support and facilitate the content recommendation process in relation to student learning styles model. This proposal also takes into account the complementarity between the learning styles, where, if a student has a tendency to a learning style, he can also learn from other learning style, thus a feature can complement other to improve learning.
\end{abstract}

Resumo. Devido à grande quantidade de objetos de aprendizagem gerados a partir de um sistema web, tem-se a necessidade de organizá-los para que os mesmos possam ser recomendados de maneira eficiente. Para isso, este trabalho propõe um agrupamento de conteúdo em grandes repositórios para apoiar e facilitar o processo de recomendação de conteúdo em relação a estilos de aprendizagem de estudantes. Esta proposta leva também em consideração a complementação entre os estilos de aprendizagem, onde um aluno que possui tendência para um estilo de aprendizagem também pode aprender com outro estilo de aprendizagem, sendo assim um característica pode complementar outra para melhorar o aprendizado.

\section{Introdução}

A área educacional tem sido um domínio de interesse para o apoio de sistemas de computação. Diante disso, diversas pesquisas passaram a ser executadas com o intuito de desenvolver e analisar Ambientes Educacionais Ubíquos (AEUs) como facilitadores de aprendizagem [Levis and Barbosa 2008]. AEUs podem empregar Objetos de Aprendizagem (OAs) como fontes de conteúdo. OAs, deste modo, são classificados como entidades passíveis de utilização dentro do processo de ensino e aprendizagem.

Outro conceito importante para a criação de sistemas de computação para ambientes educacionais são nomeados de Estilo de Aprendizagem (EA). Os EAs estão relacionados às estratégias que um aluno tende a aplicar com frequência durante sua busca por conhecimento. [Felder and Silverman 1988] criaram um modelo específico para a aprendizagem de estudantes por meio de quatro dimensões: percepção, entrada, processamento e organização. 
O primeiro passo em direção a um sistema capaz de recomendar automaticamente conteúdos de aprendizagem para estudantes é a identificação das melhores formas de organização dos OAs, de modo a permitir uma melhor qualidade na recomendação de conteúdo [Margarida et al. 2003].

Assim, utilizar algoritmos de agrupamento e classificação para organizar os OAs é uma abordagem promissora. Contudo, existem poucos trabalhos na literatura que utilizam abordagens aproveitando da clusterização dos OAs no contexto de ambientes educacionais ubíquos. Este trabalho vem suprir esta falta de abordagens com métodos de agrupamento e classificação para a organização dos OAs e analisar suas vantagens.

Uma relação entre metadados e o modelo [Felder and Silverman 1988] foi pesquisado por [Carvalho et al. 2014] apresentaram uma abordagem para a recomendação de OAs com base no mapeamento do padrão IEEE Learning Object Metadata (IEEE LOM) [IEEE 2002] com os EAs. Como resultado, foram apresentados uma quantidade mínima de campos que precisa ser utilizada para uma recomendação eficiente entre OAs.

As próximas páginas serão dividas em seções onde a Seção 2 apresenta trabalhos que estão relacionados ao tema em questão. Na Seção 3 são descritos, conceito inerente ao entendimento do trabalho. A Seção 4 descreve a abordagem utilizada e a Seção 5 mostra a conclusão sobre os estudos.

\section{Trabalhos Relacionados}

O modelo de alunos baseado nas dimensões de Felder e Silverman tem sido amplamente aplicado em diversos sistemas de recomendação encontrados na literatura. Como exemplo, Zaina et al. 2012 apresentaram a proposta E-LORS (e-Learning Object Recommendation), que se baseia nos conceitos de sistemas de recomendação, utilizando um algoritmo de filtragem baseado em conteúdo. Um requisito importante foi a utilização de OAs no padrão IEEE LOM sendo que o modelo de aluno adotado foi baseado nas dimensões de Felder e Silverman. A abordagem E-LORS não propõe a utilização de OAs agrupados como esta sendo proposto neste trabalho.

O trabalho apresentado por Paper and Mois 2009 seleciona EAs utilizando a rede neural Self Organizing Map (SOM) [Kohonen 1998]. Neste trabalho a rede recebe diferentes padrões de aprendizagem do aluno obtidos a partir de um Learning Style Questionnaire (ILSQ) no padrão de Felder e Silverman. Foi implementado o sistema Educa onde existe a presença do instrutor e dos estudantes, o instrutor cria os OAs que são classificados dentro do modelo, criando assim uma pontuação para cada objeto. O método proposto não utiliza OAs no padrão IEEE LOM para representar estilos de aprendizagem.

Uma abordagem feita por Zapata and Menendez 2009 é uma proposta para apresentar os resultados da aplicação de uma metodologia de extração de conhecimento de OAs através da utilização de quatro fontes de dados: metadados, avaliações de qualidade pedagógica, perfis de usuário, e arquivos de log de OAs em um sistemas de gestão. Este trabalho não utiliza a relação entre EAs e OAs para obter resultados.

Em um modelo de recomendação Aguiar et al. 2015 utiliza objetos de aprendizagem no padrão IEEE LOM com o modelo de Felder e Silverman a partir da filtragem de OAs com base no conteúdo e posteriormente ordena os OAs com base nos EAs do aluno e sua popularidade, desta forma a ordenação baseada em conteúdo pode deixar que objetos 
baseados em outros atributos fiquem com uma popularidade baixa. Na abordagem deste trabalho é considerado que todos atributos do OA sejam inerentes ao aprendizado.

Outra abordagem proposta por Casali et al. 2011 em encontrar OA mais adequados as suas necessidades e preferências com base em repositórios de aprendizagem distribuídos.OAs são recomendados seguindo uma abordagem baseada em regras, levando em consideração o perfil dos usuários.

Espera-se assim, que a proposta de agrupar OAs se torna uma forte contribuição para as técnicas de recomendação de conteúdo, melhorando a utilização de OAs em diferentes trabalhos, sendo uma melhoria na forma em que os objetos se organizam com base em sua similaridade.

\section{Fundamentação Teórica}

Nesta sessão, são apresentadas as definições e os conceitos necessários para o entendimento da abordagem proposta, além de citar trabalhos relacionados ao tema desta pesquisa. Serão apresentados os conceitos proposto por Felder and Silverman e o padrão utilizado para os objetos de aprendizagem. Posteriormente serão mostrados detalhes dos algoritmos utilizados no modelo.

\subsection{Estilos de Aprendizagem Felder and Silverman}

Os EAs proporcionam orientações para a individualização do ensino [Given 2002]. Até recentemente, o foco da investigação acerca dos estilos de aprendizagem centrou-se na sua aplicação ao processo de ensino e aprendizagem em cenários de sala de aula. Com o avanço nos estudos de facilitar a criação materiais educacionais multimidia e com o desenvolvimento das tecnologias baseadas na Web, a investigação acerca dos estilos de aprendizagem passou também a incluir contextos de ensino e aprendizagem em sistemas educacionais ubíquos [Levis and Barbosa 2008].

Para satisfazer a diferentes estilos é necessário que se utilize estratégias de ensino adequadas às várias perspectivas de aprendizagem. A partir desses conceitos o modelo possui quatro dimensões sendo: percepção, processamento, entrada e entendimento conceituadas a seguir respectivamente:

- Sensorial/Intuitivo: dentro do estilo sensorial é mais comum ter características praticas, concreto e exemplos, sendo que para os intuitivos é mais comum características teóricas e abstratas.

- Ativo/Reflexivo: dentro do estilo ativo é mais comum características ativas do aluno dentro do ensino, enquanto no reflexivo, o aluno é prefere materiais teóricos.

- Visual/Verbal: esta dimensão indica que um estudante pode ter preferência por informações transmitidas visualmente, sendo visual, ou informações com base em texto ou falas sendo assim verbal.

- Sequencial/Global: dentro do estilo sequencial a apresentação dos materiais deve proporcionar uma navegação preestabelecida enquanto que para a dimensão global a apresentação dos materiais deve possibilitar uma navegação por conta própria.

Este modelo é amplamente utilizado em vários trabalhos que utilizam estilos de aprendizagem [Branquinho et al. 2015, Pernas et al. 2009]. 


\subsection{Objetos de Aprendizagem}

OAs podem ser definidos como recursos digitais que são utilizados em diferentes ambientes de aprendizagem com uma base tecnológica com fins educacionais [da Silva et al. 2011]. Possuem características bem definidas conforme descrito por [Audino and da Silva Nascimento 2012] para que possam ser bem utilizados em áreas educacionais.

O modelo de dados IEEE LOM especifica quais aspectos de um OA devem ser descritos e são definidos vocabulários que pode ser utilizado para essas descrições [IEEE 2002]. Os metadados usados para descrever os OAs podem incluir informações educacionais, como forma de ensino, nível de escolaridade e pré-requisitos, além de informações a respeito do tipo de objeto, autor, proprietário entre outros.

Ele possui nove grupos distintos para representar os metadados: Geral para representar informações gerais, Ciclo de Vida onde representa histórico e estado atual do OA, Meta-metadata mostram informações sobre os metadados, Técnicos representam requisitos técnicos, Educacional possui informações relacionadas aos aspectos e educacionais, Direitos exibe os direitos, propriedade intelectual e condições de uso, Relação informa relacionamentos entre os OAs, Anotação mostra comentários sobre o uso educacional e Classificação onde possui o posicionamento do OA frente a sistemas de classificação pré-definido.

\subsection{Recomendação de Objeto}

Dentro os maiores objetivos de se utilizar OAs, a recomendação se destaca, pois permite que objetos sejam reutilizados para outras disciplinas diferentes das que foram criadas inicialmente, apesentados de forma personalizada aos estudantes. Outra característica dos objetos de aprendizagem é o fato de que pode ser disponibilizado através da internet, o que permite que um grande número de estudantes tenham acesso.

As recomendações são realizadas baseando-se em características que são relevantes ao escopo do sistema. Um sistema de recomendação com fins educacionais acompanha as ações do aluno quando este interage com determinados conteúdos para que seja possível posteriormente, sugerir materiais e serviços adequados as suas preferencias [Felfernig et al. 2007].

As recomendações devem fazer sugestões que se encaixam as expectativas dos usuários. Como por exemplo surgiram os sistemas de recomendação de [Cazella et al. 2010] em sistemas que visam auxiliar o usuário na busca e seleção de um conteúdo focado em seu perfil, funcionando como filtros de informação. Assim, o usuário terá como resultado de busca apenas os materiais mais próximos e relevantes.

\subsection{Clusterização}

Clusterização é a divisão de dados, com base na similaridade entre eles, em grupos disjuntos chamados clusters. Isso significa que dados em um mesmo cluster são mais similares do que dados pertencentes a clusters diferentes [Ochi et al. 2004]. O ato de agrupar os dados representa uma forma importante no aprendizado e compreensão.

Pode ser definido também como um problema de aprendizado não supervisionado, já que a estrutura dos dados e as propriedades que os tornam similares são desconhecidas. O objetivo da clusterização é encontrar uma organização válida e conveniente dos dados, 
ao invés de separá-los em categorias como acontece no reconhecimento de padrões e na classificação de dados [Jain and Dubes 1988]. Na Figura 1 pode-se observar as etapas de um processo de clusterização.

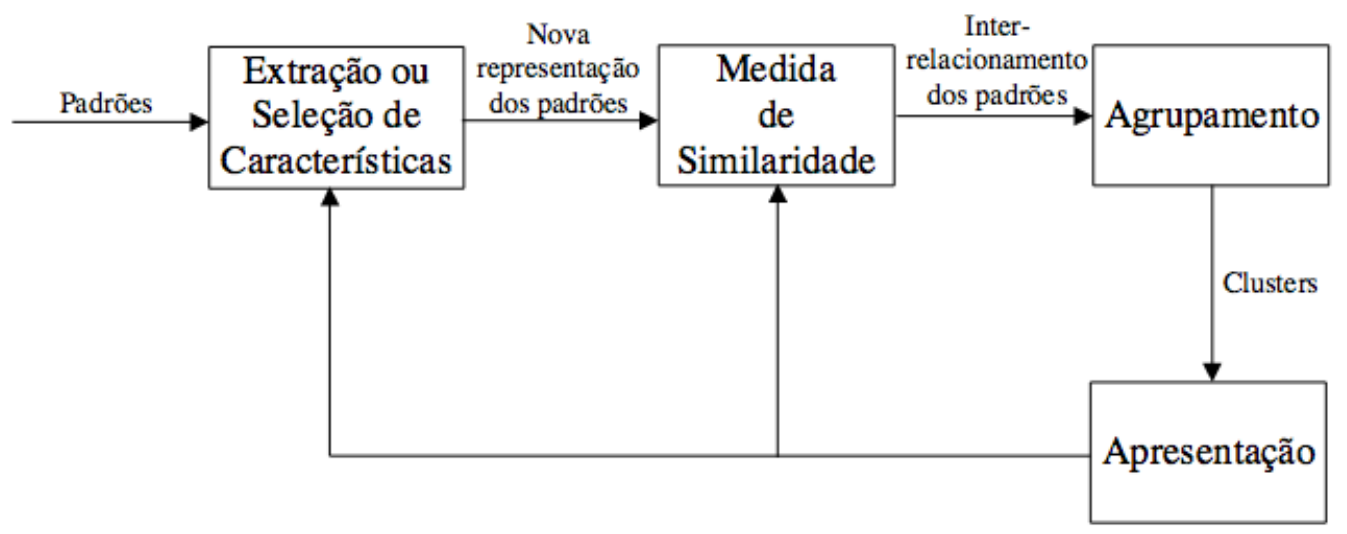

Figura 1. Etapas do processo de clusterização

As etapas apresentadas na Figura 1 são:

- Seleção de Características: a seleção de características é um processo de identificação do subconjunto mais efetivo dos atributos disponíveis para descrever cada padrão e extração de características é o uso de uma ou mais transformações nos atributos de entrada de modo a exaltar uma ou mais características dentre aquelas que estão presentes nos dados. Qualquer um ou ambas estas técnicas pode ser usado para obter um conjunto adequado de recursos para usar em agrupamentos

- Medida de Similaridade: para que a proximidade de dois dados, possa ser calculada, é necessário adotar alguma medida de similaridade entre eles. Existem diversas maneiras de quantificar a similaridade, ou dissimilaridade, entre pares de dados. A escolha da medida de similaridade adequada ao problema é fundamental para a clusterização dos dados.

- Agrupamento: define-se o modo de agrupamento dos dados, que pode ser realizado de diferentes maneiras. Os algoritmos de clusterização são classificados de acordo com as diferentes técnicas que empregam no agrupamento dos dados. Dentre esses algoritmos destacam-se os algoritmos de clusterização hierárquica e os algoritmos por particionamento. Na clusterização por particionamento o conjunto de dados é dividido em um número determinado de clusters uma única vez e no particionamento hierárquico são produzidas diversas partições do conjunto de dados com base na junção ou divisão dos clusters de acordo com a medida de similaridade.

- Apresentação/Validação: a qualidade dos clusters encontrados é avaliada sendo que essa validação pode ser feita com base em índices estatísticos ou através da comparação com outros algoritmos. Além disso, a análise dos resultados pode levar à redefinição dos atributos escolhidos e do calculo de similaridade, definidos nas etapas anteriores.

Segue alguns algoritmos de clusterização utilizados para agrupar dados. O algoritmo K-means [MacQueen et al. 1967] é um algoritmo não supervisionado muito 
utilizado em diversas áreas, sendo que a quantidade de grupos deve ser definida antecipadamente. O algoritmo funciona de maneira iterativa, onde centroides, criados inicialmente de maneira aleatória, são reajustados em direção ao centro dos grupos que são reorganizados a cada iteração.

A rede neural Self Organizing Map (SOM) [Kohonen 1998], que faz parte das redes neurais de aprendizado competitivo, é aquela na qual os neurônios de uma camada competem entre si pelo privilégio de permanecerem ativos, tal que o neurônio com maior atividade seja o único a participar do processo de aprendizado. Na rede SOM é fornecido um vetor de entrada, no qual todos os neurônios a avaliam com uma função de avaliação distinta e aquele que obtiver a maior avaliação para a entrada tem seus pesos ajustados, levando a um neurônio vencedor. Esta característica faz com que grupos vizinhos no mapa discreto possuam padrões semelhantes no espaço de entrada.

Um método também conhecido é o Density-Based Spatial Clustering of Applications with Noise (DBSCAN) [Ester et al. 1996] é um algoritmo baseado em densidade, que é significativamente efetivo para identificar agrupamentos de formato arbitrário e de diferentes tamanhos, separar os ruídos dos dados e detectar grupos padrões dentro do espaço de dados, sem qualquer informação preliminar sobre os grupos.

\section{Abordagem Proposta}

A abordagem neste trabalho é identificar grupos com características semelhantes entre os OAs, diante do conhecimento que um OA esta classificado dentro das dimensões de Felder and Silverman 1988. É considerado que uma combinação de estilos de aprendizagem (CEA) é uma tupla formada por 4 EAs, cada um pertencente a uma dimensão de Felder e Silverman sendo então possível ter 16 combinações [Dorça et al. 2013].

Um estudante que tem uma tendência forte para a característica Visual não exclui o fato que também é possível o aprendizado com uma característica Reflexiva ou Verbal. Assim este trabalho tem como desafio exibir uma proposta para a vetorização dos OAs considerando seus metadados. Assim, podemos modelar os estudantes de forma probabilística e dinâmica, que estocasticamente se enquadram a estas categorias pedagógicas ao longo do processo de aprendizagem com maior ou menor probabilidade dentro das 16 categorias possíveis.

Tabela 1. Estudante

\begin{tabular}{|lc|ccccccc}
\hline \multicolumn{8}{c}{ Estilos de Aprendizagem } \\
\hline \multicolumn{2}{|c|}{ Processamento } & Percepção & \multicolumn{2}{c|}{ Entrada } & Entendimento \\
\hline Sen. & Int. & Ati. & Ref. & Vis & Ver. & Seq. & Glob. \\
17.0 & 83.0 & 35.0 & 65.0 & 80.0 & 20.0 & 84.0 & 16.0 \\
\hline
\end{tabular}

O aluno representado pelo modelo descrito na Tabela 1, possui preferências para os estilos: intuitivo, reflexivo, visual e sequencial. Neste contexto, o maior vantagem desta abordagem é a de considerar estocasticamente todas combinação de EAs de acordo com os alunos supondo que os EAs podem estar incoerentes ou podem mudar ao longo do tempo [Dorça et al. 2013]. A proposta se baseia na teoria das cadeias de Markov (MC), que é um sistema matemático que modela as mudanças de estado entre um número finito 
Tabela 2. Relação Objetos de Aprendizagem x Felder e Silverman

\begin{tabular}{|c|c|c|c|}
\hline Structure & Interactivity Type & Learning Resource Type & Interactivity Leve \\
\hline Global & Ativo & Sensitivo & Reflexivo \\
\hline $\begin{array}{l}\text { collection } \\
\text { networked } \\
\text { hierarchical }\end{array}$ & $\begin{array}{l}\text { active } \\
\text { mixed }\end{array}$ & $\begin{array}{l}\text { simulation, graphindex, } \\
\text { table, figure, experiment, } \\
\text { self assessement }\end{array}$ & $\begin{array}{l}\text { verylow } \\
\text { low } \\
\text { medium }\end{array}$ \\
\hline Sequencial & Reflexivo & Verbal & Ativo \\
\hline $\begin{array}{l}\text { linear } \\
\text { atomic }\end{array}$ & $\begin{array}{l}\text { expositive } \\
\text { mixed }\end{array}$ & $\begin{array}{l}\text { exercise, questionnaire, } \\
\text { index, table, narrative text } \\
\text { exam, problem statement, } \\
\text { self assessement, } \\
\text { lecture }\end{array}$ & $\begin{array}{l}\text { medium } \\
\text { high } \\
\text { very high }\end{array}$ \\
\hline & & Intuitivo & \\
\hline & & diagram & \\
\hline & & Visual & \\
\hline & & $\begin{array}{l}\text { visual, diagram, figure } \\
\text { graph, experiment }\end{array}$ & \\
\hline & & Ativo & \\
\hline & & $\begin{array}{l}\text { exercise, simulation } \\
\text { questionnaire,exam } \\
\text { experiment, } \\
\text { problema statement } \\
\text { self assessment }\end{array}$ & \\
\hline & & Reflexivo & \\
\hline & & $\begin{array}{l}\text { diagram, figure, graph } \\
\text { index, slide, table } \\
\text { narrative text, lecture }\end{array}$ & \\
\hline
\end{tabular}

de possíveis estados, e eles são muitas vezes descrito por um grafo direcionado, onde as bordas são rotulados pelas probabilidades de ir de um estado para os outro [Meyn and Tweedie 2009].

A Tabela 2 representa um estudo detalhado foi realizado para relacionar os EAs de Felder e Silverman com os OAs no padrão IEEE LOM, que foi construída baseado no trabalho de Resende et al. 2014. Foi proposto um estudo dos conceitos envolvidos em ambas as teorias, a fim de pesquisar e definir os pontos em que os OAs e os EAs se relacionam. OS campos Structure, Interactivity Type, Learning Resource Type e Interactivity Level são campos que estão presentes em um OA, sendo que cada um deles possui um determinado valor, por exemplo Structure:collection que se encaixa no EA Global. Assim obteve como resultado os campos que o padrão IEEE LOM representam os EAs atendidos por determinado objeto.

Um exemplo de vetorização de um OA poderia ser representado com os atributos: Structure com atributo do tipo linear resultando em um ponto para Sequencial, Interactivity Type sendo expositive somando um ponto em Reflexivo, Learning Resource Type com o atributo table somando um ponto em Sensitivo, Verbal e Reflexivo e por final Inte- 
ractivity Level com atributo very high atribuindo um ponto em Ativo. Somando os pontos atribuídos a cada EA obtem-se o a vetorização da Tabela 3 no qual soma-se os pontos obtidos em cada EA relacionando o atributo que o ativou.

Tabela 3. Vetorização de um Objeto de Aprendizagem

\begin{tabular}{|l|l|l|l|l|l|l|l|}
\hline \multicolumn{6}{|c|}{ Objeto de Aprendizagem x Felder e Silverman } \\
\hline Ati. & Ref. & Vis. & Ver. & Seq. & Glo. & Sen. & Int. \\
\hline 1 & 2 & 0 & 1 & 1 & 0 & 1 & 0 \\
\hline
\end{tabular}

Esta vetorização possui grande importância para que possa ser utilizado os algoritmos de clusterização comentados na seção 3.4. A relevância da clusterização é alta dentro do contexto apresentado pois tem-se que um objeto de aprendizagem pode ser clusterizado baseados nos 16 cluster. Um exemplo de um cluster com base no vetor da Tabela 3 que o objeto vetorizado pertence ao cluster: Reflexivo, Verbal, Sequencial e Intuitivo.

Diante disto um OA que pode ser utilizado para recomendação de um aluno que seja Reflexivo também poderá ser recomendado para um aluno que tenha também um perfil Verbal pois as técnicas de clusterização podem criar agrupamentos para os OAs e identificar uma relação com outros grupos de OAs fazendo com que tenha uma recomendação mais dinâmica para um determinado aluno.

\section{Conclusão}

Utilizar da disponibilidade de OAs de acordo com o perfil de aprendizagem do estudante apoia o processo de ensino e aprendizagem, auxilia também o aluno, através de abordagem de conteúdos que estejam em conformidade com as suas preferencias de aprendizado.

Um ponto importante é a relação entre as preferencias definidas no modelo do aluno com as categorias descritivas dos objetos de aprendizagem do padrão LOM. $O$ relacionamento entre os EAs e os metadados dos objetos de aprendizagem permite ter uma maior concordância entre o perfil de aprendizagem identificado no aluno e os objetos de aprendizagem.

Esta abordagem proposta possibilitará os agrupamentos de OAs em grandes repositórios utilizando diferentes técnicas de agrupamentos mencionadas, sendo que será levado em questão os EAs e os metadados dos OAs. Com a vetorização e clusterização dos OAs, espera-se que a criação do processo de recomendação de OAs considerando os EAs dos estudantes. Espera-se que esta proposta permita facilitar o processo de recomendação personalizada de conteúdo considerando EAs em ambientes educacionais.

Como trabalho futuro tem como objetivo a implementação dessas técnicas no sistema Classroom eXperience [Araújo et al. 2014] para validação do modelo com estudantes reais para que possa avaliar uma melhoria em como o aluno evolui com um aprendizado baseado em suas competências.

\section{Agradecimentos}

Agradecemos a Pró-Reitoria de Pesquisa e Pós-Graduação da Universidade Federal de Uberlândia(UFU) e a Coordenação de Aperfeiçoamento de Pessoal de Nível Superior(CAPES) por todo o suporte prestado no desenvolvimento deste trabalho. 


\section{Referências}

Aguiar, J. J., Fechine ${ }^{1}$, J. M., and Costa, E. B. (2015). Recomendação de objetos de aprendizagem baseada na popularidade dos objetos e nos estilos de aprendizagem dos alunos.

Araújo, R. D., Brant-ribeiro, T., Freitas, R. S. D., Dorça, F. A., and Cattelan, R. G. (2014). Autoria automática de objetos de aprendizagem a partir de captura multimídia e associação a estilos de aprendizagem. (Cbie):229-238.

Audino, D. F. and da Silva Nascimento, R. (2012). Objetos de aprendizagem-diálogos entre conceitos e uma nova proposição aplicada à educação. Revista Contemporânea de Educação, 5(10).

Branquinho, A., Lopes, C., Dorça, F., Fernandes, M., Cattelan, R., and de Ávila, A. J. N. (2015). Aquisição automática de competências num ambiente educacional ubíquo. 26(1):1207.

Carvalho, V. C., Dorça, F. A., Cattelan, R. G., and Araújo, R. D. (2014). Uma abordagem para recomendaç ao automática e dinâmica de objetos de aprendizagem baseada em estilos de aprendizagem.

Casali, A., Gerling, V., Deco, C., and Bender, C. (2011). A recommender system for learning objects personalized retrieval. Educational Recommender Systems and Technologies: Practices and Challenges, pages 182-210.

Cazella, S. C., Nunes, M., and Reategui, E. (2010). A ciência da opinião: Estado da arte em sistemas de recomendação. JAI Jorn. Atualização em Informática da SBC. Rio Janeiro, RJ PUC Rio, pages 161-216.

da Silva, E. L., Café, L., and Catapan, A. H. (2011). Os objetos educacionais, os metadados e os repositórios na sociedade da informação. Ciência da Informação, 39(3).

Dorça, F. A., Lima, L. V., Fernandes, M. A., and Lopes, C. R. (2013). Automatic student modeling in adaptive educational systems through probabilistic learning style combinations: a qualitative comparison between two innovative stochastic approaches. Journal of the Brazilian Computer Society, 19(1):43-58.

Ester, M., Kriegel, H.-P., Sander, J., and Xu, X. (1996). A density-based algorithm for discovering clusters in large spatial databases with noise. In $K d d$, volume 96, pages 226-231.

Felder, R. M. and Silverman, L. K. (1988). Learning and teaching styles in engineering education. Engineering education, 78(7):674-681.

Felfernig, A., Friedrich, G., and Schmidt-Thieme, L. (2007). Guest editors' introduction: Recommender systems. IEEE Intelligent Systems, (3):18-21.

Given, B. (2002). The overlap between brain research and research on learning style. In Learning Styles: Realibility \& Validity, Proceedings of the 7 th Annual ELSIN Conference, pages 173-178.

IEEE (2002). IEEE Standard for Learning Object Metadata.

Jain, A. K. and Dubes, R. C. (1988). Algorithms for clustering data. Prentice-Hall, Inc. 
Kohonen, Teuvo, P. (1998). Self-organizing maps of symbol strings. Neurocomputing, 21(1):19-30.

Levis, D. and Barbosa, J. L. V. (2008). Aperfeiçoamento Automático do Perfil do Aprendiz.

MacQueen, J. et al. (1967). Some methods for classification and analysis of multivariate observations. In Proceedings of the fifth Berkeley symposium on mathematical statistics and probability, volume 1, pages 281-297. Oakland, CA, USA.

Margarida, L., Tarouco, R., and Fabre, M.-c. J. M. (2003). Reusabilidade de objetos educacionais. pages $1-11$.

Meyn, S. and Tweedie, R. L. (2009). Markov chains and stochastic stability. prologue by peter w. glynn. cambridge mathematical library.

Ochi, L. S., Dias, C. R., and Soares, S. S. F. (2004). Clusterização em mineração de dados. Instituto de Computação-Universidade Federal Fluminense-Niterói.

Paper, C. and Mois, J. (2009). A Kohonen Network for Modeling Students ' Learning Styles in Web 2 0 Collaborative Learning Systems. (NOVEMBER).

Pernas, A. M., Gasparini, I., de Oliveira, J. P. M., and Pimenta, M. S. (2009). Um ambiente ead adaptativo considerando o contexto do usuário (position paper). pages 1151-1156.

Resende, D. T., Dorça, F. A., Cattelan, R. G., and Rafael, D. (2014). Em direção à recuperação automática de objetos de aprendizagem em repositórios através da associação dos estilos de aprendizagem de estudantes com metadados no padrão IEEE-LOM. (Cbie):445-454.

Zaina, L. A. M., Cardieri, M. A. C. A., Engenharia, F. D., and Sorocaba, D. (2012). e-LORS : Uma Abordagem para Recomendação de Objetos de Aprendizagem. 20.

Zapata, A. and Menendez, V. H. (2009). Discovering Learning Objects Usability Characteristics. 